\title{
Blind Separation of Underwater Acoustic Signals
}

\author{
N. Benchekroun, and A. Mansour, Member, IEEE
}

\begin{abstract}
Acoustic tomography is an issue of great importance in many applications. Few algorithms have been dedicated to the passive acoustic tomography of a single input single output (SISO) channel. As matter of fact, most of those algorithms can not be applied in a real situation i.e. for a Multi-Input MultiOutput (MIMO) channel. In this paper, we discussed at first the features of the acoustic channel and signals then we proposed an architecture to separate acoustic signals issued from an acoustic realistic channel. The proposed structures used algorithms based on independent component analysis (ICA). We should mention here that many algorithms have implemented and tested but only two algorithms give good results. The latter algorithms minimize two different second order statistic criteria in the frequency domain. Finally, some simulations have been presented and discussed.
\end{abstract}

\section{INTRODUCTION}

To estimate ocean physical parameters (such as temperature distribution, currents, sediment structure), The Ocean Acoustic Tomography (OAT) is widely used. Acoustic tomography is used in many civil or military applications such as: Mapping underwater surfaces, meteorological applications, Warfare. OAT basic principle relies on dependence of the acoustic propagation on the spatial distribution of the ocean parameters, in particular temperature. Two kind of OAT can be used: Classical active OAT and Passive ones. In active OAT, a typical sound source in known fixed position should be emitted. At the receiver, one can estimate the channel parameters by using the properties of the received signal. Many algorithms [11, 12] have been developed to deal with active acoustic tomography.

Recently, the Passive Acoustic Tomography (PAT) [10] which is an acoustic tomography variant where the usual cooperative acoustic source is replaced by a non-cooperative noise source as for example a ship of opportunity, has taken an increased importance because it exhibits three main advantages: suits the Submarine Acoustic Warfare Applications, avoids the need of both economically and operationally expensive signal sources and allows the investigation of areas as wide as those swept by the moving ships and finally doesn't perturb the ecological system. In typically real world PAT applications, underwater signals are generated by various sources in motion whose number's and positions are hardly identified. With more than two sources, many actual tomography algorithms can't give pleasant results. Many others don't work well or at all when the emitted signals are large band [14].
The Blind Separation of Sources (BSS) problem consists on retrieving unknown mixed independent sources from their observed mixture. To reach that goal, researchers use Independent Component Analysis (ICA) methods. Actually, the last methods are ones of the most up-to-date methods in signal processing. These methods can be applied in many fields including speech processing, data communication, biomedical signal processing, radar, sonar as well as the surveillance and control of airport and sea traffic. In this paper, we apply BSS algorithms in PAT in order to improve and simplify the PAT algorithms as well as the processing of the received signals.

\section{ACOUSTIC CHANNEL MODEL AND ACOUSTIC SIGNALS}

\section{A. Acoustic Channel Model}

Under some mild assumptions [12], acoustic submarine channel can be considered as a multiple paths which, in frequency domain, each of them can be defined by a complex constant gain (i.e. a real lag $\tau_{i}$ and a real gain $\mathrm{C}_{\mathrm{i}}$ ).

For a classic Single Input Single Output (SISO) Channel, the relationship between the emitted signal $s(t)$, which will be called later on as source signal and the received (or observed signal $x(t)$ is given by:

$$
x(t)=\sum_{i=0}^{M} C_{i} s\left(t-\tau_{i}\right)+n(t)
$$

Where $n(t)$ stands for an Additive White Gaussian Noise (AWGN) and $\mathrm{M}$ is the channel order or the number of paths. In general case, the channel can be considered as Multiple Input Multiple Output (MIMO) channel whose mathematical model is given by the following equation:

$$
X(n)=\sum_{i=0}^{M} H(i) S(n-i)+N(n)
$$

Where $X(n)$ is the $q \times 1$ vector of observed signals, $S(n)$ is the $p \times 1$ vector of sources, N(n) is a AWGN $q \times 1$ vector and $\mathrm{H}$ is the mixing system where $\mathrm{H}(\mathrm{z})=(\mathrm{hij}(\mathrm{z}))$ is a $q \times p$ complex polynomial matrix. In the following, we assume that the channel is a linear and causal one and that the coefficients hij(z) are FIR filters.

It is obvious that for PAT applications, Blind Source Separation (BSS) problem can be very helpful. In BSS terminology, the above problem can be solved by 
considering the blind separation of linear mixed signals. Linear mixtures include the following two models:

- Convolutive Mixtures: When the channel is a multi-path with memory channel (i.e. an echo real channel). This type of mixture can represent the general case of acoustic underwater channel, see equation (2).

- Instantaneous Mixtures: this model is a simplified convolutive one, especially when the channel is a memoryless channel (without echo). The last case can be observed in deep water environment. Then, equation (2) can be rewritten as:

$$
X(t)=H S(t)+N(t)
$$

In our study, it is the general case (2) which will be considered. The instantaneous case has been studied in [1]. Convolutive mixture algorithms are generally time consuming algorithms. Few of them have been developed in the literature [13] and are dedicated to specific tasks and signals. In our knowledge, none of them has been optimized to deal with our problem.

In practise, our simulated underwater acoustic channel used the ray theory as a propagation model which is the more appropriate model to our application. In our model, a sand bottom had been considered and some random coefficients have been added to characterize varieties on the top and the bottom of the channel. Finally, an acoustic model proposed in [15] was used to consider the acoustic propagation effect.

\section{B. Acoustic Signals}

Generally, Independent Component Analysis (ICA) algorithms use only the independence assumption of the sources. In PAT applications, the sources are some signals of opportunities. Extensive experimental studies have been conducted by a research engineer in our laboratory to classify and characterize many recorded artificial (made by human activities as boats, ships or submarine noises,etc.) and natural (mainly animals sounds or noises) signals. That study is of extreme important to ours. In fact, according to the characterization study, one can conclude the following facts:

- All the recorded signals have a background ocean noise which can be considered as an Additive White Gaussian Noise (AWGN).

- Many signals are Gaussians or close to Gaussians ones.

- Even if all the signals are non-stationary signals, some of them have more or less periodic components as boat noises.

- Natural signals are very sparse ones and artificial ones are very noisy.

The above mentioned properties were very useful to select appropriate ICA algorithms.

\section{SEPARATION STRUCTURES APPLIED TO ACOUSTIC SIGNALS}

\section{A. Background and Assumptions}

The selected algorithms have been tested using the following three steps:

- To valid our implementation, we use same (or similar) signals used by the authors of the algorithm, and we try to obtain same (or similar) results shown by the authors.

- After that, the same algorithm should be tested on simple mixture of acoustic signals.

- At the end, we try the algorithm on real signals which cross our simulated underwater acoustic channel.

In our experimental studies, we found that at the third step none of the tested algorithms can unfortunately achieve a satisfactory separation according to a set of performance indexes [16]. For this reason, a complete separation structure has been implemented using the following pre- and postprocessing modules of the signals see Fig. 1:

- The Low Pass Filter helps us to reduce the impact of AWGN.

- The filter bank is to improve the frequency resolution of the frequency algorithms.

- The recovering module of the signals is based on second order statistics and it uses the correlation of the signals in time or frequency domain.

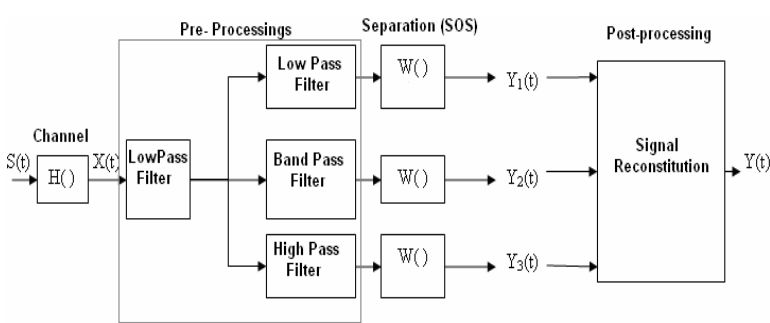

Fig.1. The proposed structure

Using these pre- and post-processings, we found that among the tested algorithms, only two [2,7] have given satisfactory results. They were dedicated to separate non-stationary sources (audio or music signals) and will be called in the following SOS [2] and Parra [7]. Both of them are implemented in frequency domain and are using discrete frequency adapted filter.

\section{B. A Frequency Domain Method For Blind Source Separation Of Convolutive Audio Mixture (SOS)}

$\mathrm{K}$. Rahbar et al. in [2, 6] propose an algorithm which minimize a criterion based on the cross-spectral density matrix of the observed signals. For non-stationary signals, the latter matrix depends of frequency and time epoch $\mathrm{m}$ : Let $P_{x}(\omega, m)$ represent the cross-spectral density matrix of the observed signal at frequency $\omega$ and time epoch $\mathrm{m}$. Based 
on the above assumptions, the main idea of this approach comes from the following equation:

$$
P_{x}(\omega, m)=H(\omega) P_{s}(\omega, m) H^{+}(\omega)+\sigma^{2} I
$$

Where $P_{s}(\omega, m)$ is a diagonal matrix which represents the cross-spectral density matrix of the sources at epoch $\mathrm{m}$ and $\sigma^{2}$ is the power of $\mathrm{N}(\mathrm{t})$. In practise, $\omega$ has to be discretized as $\omega_{k}=2 \pi k / K$ where $\mathrm{K}$ is the total number of frequency samples. For $\mathrm{q}>\mathrm{p}, \sigma^{2}$ can be estimated from the smallest eigenvalue of the matrix $\tilde{P}_{x}(\omega, m)$. Therefore, we consider the following noise free case:

$$
P_{x}(\omega, m)=H(\omega) P_{s}(\omega, m) H^{+}(\omega)
$$

To do so, the authors of [2] developed a two stages algorithm. The first stage employs joint diagonalization [3, $4,5]$ of the set of cross power spectral density matrices

$P_{x}(\omega, m), \mathrm{m}=0, \ldots, \mathrm{M}-1$ at each frequency $\omega_{k}$, over $\mathrm{M}$ epochs, to estimate the mixing system up to a permutation and diagonal scaling ambiguity at each frequency bin. In [2], the authors used the following least-squares based joint diagonalization criterion for the case when a sample estimate

of each $P_{x}(\omega, m)$ is available:

$$
\min _{B\left(\omega_{k}\right), \Lambda(m) 1} \sum_{k=0}^{K-1} \sum_{m=0}^{M-1}\left\|\hat{P}_{x}\left(\omega_{k}, m\right)-B\left(\omega_{k}\right) \Lambda\left(\omega_{k}, m\right) B^{+}\left(\omega_{k}\right)\right\|_{F}^{2}
$$

Where $B\left(\omega_{k}\right)$ is an estimate of the mixing system $H\left(\omega_{k}\right)$, $P_{x}\left(\omega_{k}, m\right)$ is a sample estimate of the observed signal cross spectral density matrix at frequency bin $\omega_{k}$ and time epoch $\mathrm{m}$, and $\Lambda\left(\omega_{k}, m\right)$ is a diagonal matrix, representing the unknown cross-spectral density matrix of the sources at each epoch $\mathrm{m}$. In the second stage of the algorithm, the authors propose a novel solution for solving the permutation problem which exploits the cross-frequency correlation between diagonal values of $\Lambda\left(\omega_{k}, m\right)$ and $\Lambda\left(\omega_{k+1}, m\right)$. Finally, having $B\left(\omega_{k}\right)=H\left(\omega_{k}\right) \Pi \mathrm{D}\left(\omega_{k}\right)$ at each frequency bin $\left(\Pi \in R^{p \times p}\right.$ is a permutation matrix and $\mathrm{D}\left(\omega_{k}\right)$ represents a frequency-dependent diagonal matrix), we can calculate the separating matrix $W\left(\omega_{k}\right)$ from the following equation:

$$
W\left(\omega_{k}\right)=B^{+}\left(\omega_{k}\right)
$$

Where $B^{+}\left(\omega_{k}\right)$ is the pseudo inverse of matrix $B\left(\omega_{k}\right)$.

\section{Convolutive Blind Separation of Non-Stationary Sources (Parra)}

The main idea of the algorithm [7] proposed by L. Parra and C. Spence is similar to the previous ones [2] and [8]. This algorithm will be called later "Parra". The main difference between the two approaches remains on the considered estimation model. The authors prefer instead of estimating a forward model $\mathrm{B}$ of $\mathrm{H}$ and finding a stable inverse to directly estimate a stable multi-path backward FIR model $\mathrm{W}$. They wish to find model sources with cross-powerspectral-density satisfying:

$$
\hat{\Lambda}_{s}(\omega, m)=W(\omega)\left[\hat{P}_{x}(\omega, m)-\Lambda_{n}(\omega, m)\right] W^{H}(\omega)
$$

A multipath model $\mathrm{W}$ that satisfies these equations for $\mathrm{M}$ epochs simultaneously can be found with a Least Square

$$
\text { estimate: } \hat{W}, \hat{\Lambda}_{s}, \hat{\Lambda}_{n}=\underset{\substack{W, \Lambda_{s}, \Lambda_{n} \\ W(\tau)=0, \tau>Q<<K \\ W_{i i}(\omega)=1}}{\arg \min } \sum_{k=1}^{K} \sum_{m=1}^{M}\|\operatorname{Er}(\omega, m)\|^{2}
$$

Where $\operatorname{Er}(\omega, m)=W(\omega)\left[P_{x}(\omega, m)-\Lambda_{n}(\omega, m)\right] W^{H}(\omega)-\Lambda_{s}(\omega, m)$

The additional time domain constraint on the filter size Q of $\mathrm{W}$ relative to the frame size $\mathrm{K}$, i.e. $W(\tau)=0 \quad \forall \tau>Q<<K$ will restrict the solutions to be continuous in the frequency domain and solve the frequency permutation problem. The same idea was used in [9].

\section{EXPERIMENTAL RESULTS}

Many simulations have been conducted. Generally, we need over 600000-1200000 samples to achieve the separation. The original sources are sampled at $44 \mathrm{KHz}$. In almost all the simulations, the separation of artificial or natural mixtures have been successfully achieved. In these simulations, we have set the channel depth between 100 to $500 \mathrm{~m}$, the distances among the sources or the sensors are from 30 to $300 \mathrm{~m}$, the distances among the different sources and the diverse sensors are from 1500 to $2500 \mathrm{~m}$, the number of sensors ( 3 in all the simulations) is strictly greater than the number of sources (2).

Fig. 2 represents the experimental results obtained by applying the structure (SOS) proposed in fig.1 to separate a mixture of acoustic signals (Whale and ship). We should mention here, that good results have been obtained by only applying SOS algorithm except for some configurations notably when the sources are close to the water surface. For the latter cases, we found that the Parra algorithm before SOS algorithm could improve the overall results.
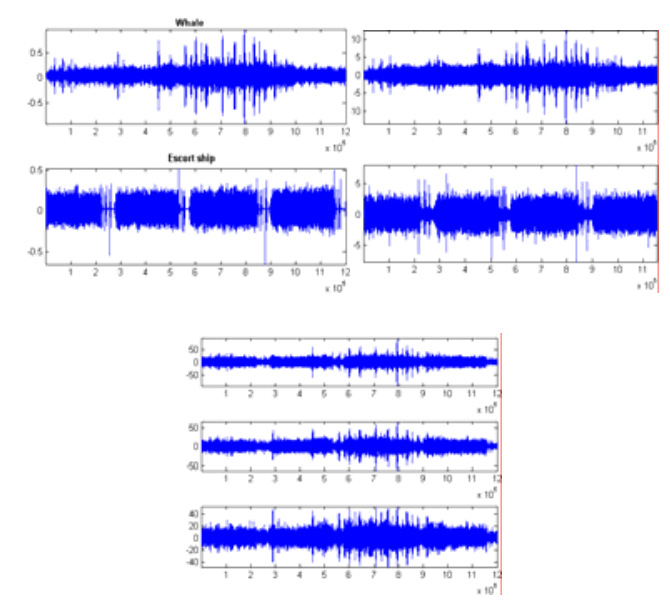
Fig. 2. Experimental results: the first line shows respectively the original and the estimated sources, the second line contains the observed signals (the sources are: a whale sound and a boat noise)

Best results have been obtained when both algorithms Parra and SOS are used and the number of sensors is strictly greater than the number of sources, as shown in Fig3.

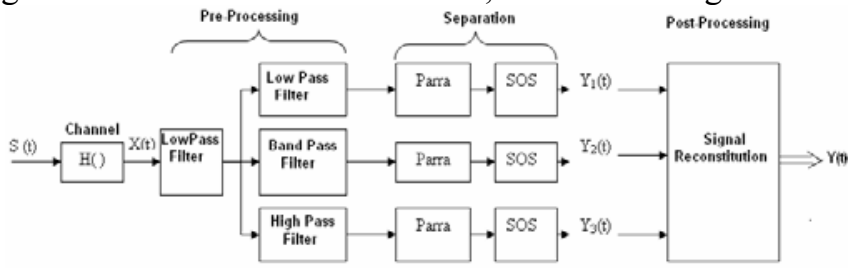

Fig.3. The general structure

Fig. 4 shows the experimental results obtained by applying the general structure (Parra+SOS) proposed in fig.3 to separate a mixture of acoustic signals (two ships) convolved in an acoustic channel.

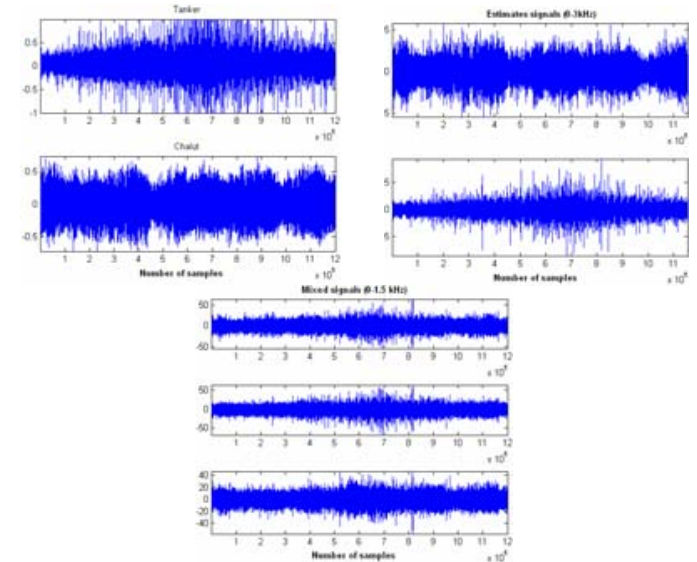

Fig. 4. Experimental results: The figure shows respectively the original, the estimated sources at the first line and the observed signals at the second line (the sources are: two boat noises).

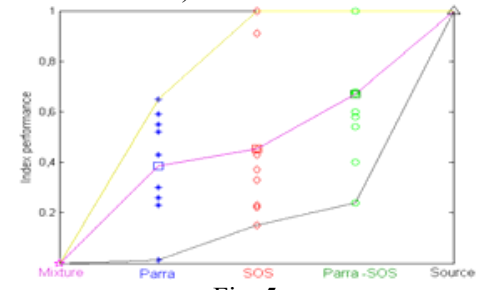

Fig. 5

Fig. 5 shows the experimental results obtained by the different algorithms (Parra, SOS or Parra + SOS) applied to the same acoustic sources and the same configuration of the acoustic channel at each simulation. In this figure, a normalized performance index based on nonlinear decorrelation is used [16]. This index is forced to be zero for the mixture values and 1 for the sources.

\section{CONCLUSION}

In this paper, we presented a general structure using BSS algorithms applied on a real word application which is the Passive Acoustic Tomography (PAT). After many simulations, we obtained experimental results that showed the necessity of considering pre-processing and post processing which have to be applied to the observed signals in order to achieve properly the separation of the sources. Many algorithms have been implemented and tested on our application but only few BSS algorithms dedicated to the separation of non-stationary signals gave satisfactory results. Our future work consists on developing a BSS algorithm which can use other features of acoustic signals.

\section{REFERENCES}

[1] Mansour, C. Gervaise, "ICA applied to passive oceanic tomography," WSEAS Trans. Acoustics and Music, Issue 2, Vol. 1, April 2004.

[2] K.Rahbar, James P. Reilly, "A new frequency domain method for blind source separation of convolutive audio mixture," IEEE trans. Speech and audio processing, 2005.

[3] B.Flurry, W.Gautschi, "An algorithm for the simultaneous orthogonal transformation of several positive definite symmetric matrices to nearly orthogonal," SIAM, vol.7, pp. 169 -184, 1986.

[4] J.F. Cardoso, A. Soulamic, "Blind Beamforming for non gaussian Signals," IEEE proceedings - F, page 362- , vol. 140, n 6, Dec. 93

[5] Belouchrani, K. Abed-Meraim, J.F. Cardoso, "A blind separation technique using second order statistics," IEEE on Trans. Signal Processing, vol. 45, pp. 434 - 444, Feb. 1997.

[6] K. Rahbar, J. Reilly, H. Manton, "Blind identification of MIMO FIR systems driven by quasi-stationary sources using second order statistics: A frequency domain approach," IEEE Trans. on Signal Processing, vol. 52, pp. 406 - 417, February 2004.

[7] L. Parra, C. Spence, "Convolutive blind separation of non-stationary sources," IEEE Trans. Speech and Audio Processing, vol. 8, No. 3, May 2000

[8] Capdevielle, C. Serviere, J.L. Lacoume, "Blind separation of wideband sources in the frequency domain," In Proc. ICASSP 95, pp. 2080-2083, 1995.

[9] Mansour, M. Kawamoto, C. Puntonet, "A time-frequency approach to blind separation of under-determined mixture of sources," International Conference of Applied Simulation and Modelling, September 3-5, 2003, Marbella, Spain.

[10] D.Gaucher, C. Gervaise, G. Jourdain, "Feasability of passive oceanic acoustic tomography in shallow water context: Optimal design of experiments," In European Conference on Under-water Acoustics ECUA 2004, pages 56-60, Delft, Netherlands, 5-8 July 2004.

[11] Gervaise, A. Quinquis, I. Luzin, "High resolution identification of an underwater channel from unknown transient stimuli," In 18eme Colloque Gresti, Toulouse, 10-13 Sept 2001.

[12] Gervaise, A. Quinquis, N. Martins, "Time frequency approach of blind study of acoustic submarine channel and source recognition," In Physics in Signal and Image Processing, PSIP 2001, Marseille, France, January 2001.

[13] Mansour, A. Kardec Barros, N. Ohnishi, "Blind separation of sources: Methods, assumptions and applications," IEICE Transactions on Fundamentals of Electronics, Communications and Computer Sciences, E83-A(8):1498-1512, August 2000.

[14] N. Martins, S. Jesus, C. Gervaise, A. Quinquis, "A time-frequency approach to blind deconvolution in multipath underwater channels," In Proceedings of International Conference on Acoustics Speech and Signal Processing 2002, ICASSP2002, Orlando, Florida, U.S.A, 1317 May 2002.

[15] M. Shulkin and H. W. Marsh, "Sound absorption in sea water," Journal of the Acoustical Society of America, vol. 134, pp. 864-865, 1962.

[16] Mansour, "A survey of real world performance indexes of ICA algorithms," in preparation 2006. 